\title{
Complex nonequilibrium dynamics of stacked polystyrene films deep in the glassy state
}

\author{
Virginie M. Boucher, ${ }^{1}$ Daniele Cangialosi, ${ }^{1, a)}$ Angel Alegría, ${ }^{1,2}$ \\ and Juan Colmenero ${ }^{1,2,3}$ \\ ${ }^{1}$ Centro de Fsica de Materiales (CSIC-UPV/EHU), Paseo Manuel de Lardizabal 5, 20018 San Sebastián, Spain \\ ${ }^{2}$ Departamento de Fisica de Materiales, Universidad del Pais Vasco (UPV/ EHU), Apartado 1072, \\ 20080 San Sebastián, Spain \\ ${ }^{3}$ Donostia International Physics Center, Paseo Manuel de Lardizabal 4, 20018 San Sebastián, Spain
}

(Received 14 November 2016; accepted 10 February 2017; published online 1 March 2017)

\begin{abstract}
We investigate the kinetics of enthalpy recovery in stacked glassy polystyrene (PS) films with thickness from 30 to $95 \mathrm{~nm}$ over a wide temperature range below the glass transition temperature $\left(T_{g}\right)$. We show that the time evolution toward equilibrium exhibits two mechanisms of recovery, in ways analogous to bulk PS. The fast mechanism, allowing partial enthalpy recovery toward equilibrium, displays Arrhenius temperature dependence with low activation energy, whereas the slow mechanism follows pronounced super-Arrhenius temperature dependence. In comparison to bulk PS, the time scales of the two mechanisms of recovery are considerably shorter and decreasing with the film thickness. Scaling of the equilibration times at various thicknesses indicates that the fast mechanism of recovery is compatible with the free volume holes diffusion model. Conversely, the slow mechanism of recovery appears to be accelerated with decreasing thickness more than predicted by the model and, therefore, its description requires additional ingredients. The implications, from both a fundamental and technological viewpoint, of the ability of thin polymer films to densify in relatively short time scales are discussed. Published by AIP Publishing. [http://dx.doi.org/10.1063/1.4977207]
\end{abstract}

\section{INTRODUCTION}

Liquids maintained in the metastable supercooled state below their melting temperature and further cooled down undergo the so-called glass transition. This occurs in a temperature range that is usually characterized by a single temperature: the thermal glass transition temperature, $T_{g}$, which depends on the applied cooling rate. ${ }^{1,2}$ An abrupt increase of the rate of spontaneous fluctuations is observed when cooling the supercooled liquid toward $T_{g}$. This temperature marks the crossover from the metastable supercooled state to the nonequilibrium glass and, thereby, can be viewed as an equilibrium to nonequilibrium transition. In the glassy state, the system slowly evolves toward equilibrium, a phenomenon known as physical aging ${ }^{3}$ or structural recovery. ${ }^{4}$ Conventionally, it is assumed that the $T_{g}$ and the kinetics of equilibrium recovery are one-to-one related to the rate of spontaneous fluctuations, a fact that has been demonstrated in bulk systems. ${ }^{5-8}$ However, it has to be pointed out that these two aspects of glassy dynamics are in principle different from a conceptual viewpoint. ${ }^{9,10}$ In particular, while the rate of spontaneous fluctuations represents an intrinsic property of the unperturbed system, measuring the $T_{g}$ or the kinetics of equilibrium recovery implies the application of perturbations larger than the amplitude of spontaneous fluctuations.

The last two decades have seen a growing interest on how the phenomena associated with the glass transition are

\footnotetext{
a)Electronic mail: daniele.cangialosi@ehu.eus.
}

modified when one or more dimensions are reduced to the nanoscale. ${ }^{11-14}$ Within this framework, thin polymer films have been by far the most investigated geometry. Nowadays, there exist numerous evidences showing that deviations from bulk behavior of the nonequilibrium dynamics, that is, decrease of $T_{g}$ (Ref. 15) and acceleration of equilibrium recovery in the physical aging regime, ${ }^{16,17}$ appear at film thicknesses considerably larger than those where alterations of the rate of spontaneous fluctuations are observed. ${ }^{18-20}$ Such decoupling has been observed measuring simultaneously the rate of spontaneous fluctuations and the thermal $T_{g} \cdot{ }^{21-24}$ Additional indications can be provided by experiments where the same perturbation is applied in the linear and non-linear regimes, for instance in calorimetry where the perturbed magnitude is the entropy. In the former case, thin polymer films exhibit essentially bulk-like rate of spontaneous fluctuations down to thickness of $\sim 10 \mathrm{~nm},{ }^{22,25,26}$ whereas significant deviations from the bulk $T_{g}$ are observed at similar thicknesses and observation time scales. ${ }^{27,28}$ In any case, effects on the rate of spontaneous fluctuations in nanostructured polymers are limited to the very first layers close to the interface. ${ }^{18-20} \mathrm{Con}$ versely, deviations of the nonequilibrium dynamics can be visible for length scale of nanostructuring as large as microns, as shown in films ${ }^{17,29-33}$ and polymer nanocomposites. ${ }^{34-37} \mathrm{~A}$ plausible explanation to the more pronounced effect of reducing film thickness on the nonequilibrium dynamics relies on purely geometric arguments, in particular on the role of "free" interfaces. ${ }^{38-40}$

Recently, monitoring physical aging of several polymers deep in the glassy state over times scales as large as one year, 
we have shown that the enthalpy recovery exhibits two decays: one with partial ${ }^{41}$ and the other with complete recovery of equilibrium. ${ }^{42}$ This suggests the existence of two molecular mechanisms drawing the polymer glass toward equilibrium; a result that, for instance, can be rationalized within the Random First Order Transition Theory (RFOT). ${ }^{43}$ A similar behavior was shown by Wimberger-Friedl and de Bruin on glassy polycarbonate, measuring the volume recovery at room temperature for several years. ${ }^{44}$ These results indicate that long aging times are required to observe the presence of the two mechanisms of recovery in bulk polymers. Conversely, when the recovery behavior is monitored for relatively short time scales, only one decay is generally observed..$^{45,46}$ In such a case, partial recovery of equilibrium is well documented in a large number of works. These are reviewed in Ref. 10. One exception in this sense is represented by enthalpy recovery studies over long aging time on a polystyrene (PS) with wide distribution of molecular weights, where only one decay has been observed. ${ }^{47}$ Such wide distribution may be responsible for the absence of the two steps decay, an aspect that needs to be explored in the future.

Analysis of the temperature dependence of the time to reach each plateau showed that the slow mechanism of recovery exhibits super-Arrhenius temperature dependence. Conversely Arrhenius temperature dependence with low activation energy was found for the fast mechanism of recovery. Similar experiments were recently conducted in $30 \mathrm{~nm}$ thick PS films. ${ }^{48}$ These also revealed the presence of two mechanisms of equilibrium recovery. Moreover, in this case, the fast one was found to be effective in inducing significant densification at temperatures far below $T_{g}$, a fact that was exploited to achieve low energy glasses.

Apart from polymer glasses, physical aging experiments on other types of glasses - in particular glycerol ${ }^{49}$ and chalcogenide glasses, ${ }^{50,51}$ showing the presence of multiple decays - point toward the universality of such behavior. The presence of two mechanisms of equilibration in the glassy state is indicated by specific heat measurements in metallic glasses. ${ }^{52,53}$ Furthermore, recent studies on the relaxational behavior of metallic glasses showed the presence of a weakly activated molecular mechanism deep in the glassy state. $^{54,55}$

In this work, we extend the enthalpy recovery behavior of thin polystyrene (PS) films to thicknesses in the range $30-95 \mathrm{~nm}$. In analogy to bulk PS, we find that such films also exhibit two mechanisms of equilibrium recovery. However, given the ability of thin films to equilibrate faster than bulk PS, thin films show such behavior in considerably shorter time scales. As a result, the fast mechanism of recovery, exhibiting mild temperature dependence, allows massive enthalpy recovery. This implies that films aged in appropriately chosen annealing conditions are able to explore thermodynamic states with low energy, otherwise inaccessible in reasonable time scales in bulk glasses. These results are discussed in terms of the mechanisms driving glasses toward equilibrium. Furthermore, given the high density reachable in appropriate annealing conditions, the outcome of the present study is discussed in light of the possible implications in a diverse range of applications.

\section{EXPERIMENTAL}

\section{A. Materials and samples preparation}

Linear polystyrene (PS) $\left(M_{w}=1408 \mathrm{Kg} / \mathrm{mol}\right.$ and $M_{w} / M_{n}$ $=1.17$ ) was purchased from Polymer Source, Inc. Before spin coating under the fume hood onto ultraflat glass slips $(18 \times 24 \mathrm{~mm}$; Menzel-Glaser) at $50 \mathrm{rpm}$, during $30 \mathrm{~s}$, PS was dissolved in toluene at variable concentrations to control the film thickness. As-deposited PS films were annealed in vacuum at $433 \mathrm{~K}$ for 4 days in oil-free oven to remove the solvent and the stress induced by spin coating. ${ }^{56}$ The sample thickness was checked by atomic force microscopy (AFM), which also revealed the absence of inhomogeneities in the films. Films with thicknesses 30,67, and $95 \mathrm{~nm}$ were considered in the present study. As-prepared films were subsequently removed from the glass slips by floating in water, and directly transferred into pans for differential scanning calorimetry (DSC) until a weight of $1-5 \mathrm{mg}$ was reached. A sample of bulk PS was also prepared with similar mass as the thin film samples to perform control experiments (see the supplementary material). All samples were dried in oven under vacuum at $353 \mathrm{~K}$ for one day to remove any trace of superficial water from the process of floating.

\section{B. DSC measurements}

Specific heat measurements were performed by DSCQ2000 calorimeter from TA-Instruments, calibrated with melting indium. The cooling system consisted of the DSC refrigerated cooling system by TA instruments. Several experiments were reproduced in another DSC-Q2000 calorimeter. In both cases, the nitrogen line uses a highly dried gas, obtained from evaporation of liquid nitrogen. In all cases, a nitrogen flow was warranted during the entire duration of annealing experiments in the glassy state and the preceding/subsequent thermal cycles. All annealing experiments were carried out inside the calorimeter cell. All experiments began heating up the samples at $393 \mathrm{~K}$ and keeping the samples for $1 \mathrm{~min}$ at this temperature. Such temperature and time scale are large enough to remove the previous thermal history. At the same time, given the very high molecular weight employed in the present study, this temperature is low enough to avoid the interdiffusion between chains belonging to different films, which would destroy the films nanostructure ${ }^{57}$ Samples were subsequently cooled down at $20 \mathrm{~K} / \mathrm{min}$ to $233 \mathrm{~K}$ before stabilizing at $20 \mathrm{~K} / \mathrm{min}$ at the selected aging temperature, $T_{a} \geq 233 \mathrm{~K}$. Samples were aged at $T_{a}$ for times ranging from minutes to several weeks and subsequently cooled to $183 \mathrm{~K}$ at a cooling rate of $20 \mathrm{~K} / \mathrm{min}$, prior to reheating at $10 \mathrm{~K} / \mathrm{min}-$ $393 \mathrm{~K}$ for data collection. Reference scans, that is, those where samples were cooled down and heated up immediately after, were also carried out both before and after the aging cycle. The two scans overlap for all of our measurements.

The amount of recovered enthalpy during aging at temperature $T_{a}$ for a given aging time $t_{a}$ was evaluated from the difference between the integral of the scan recorded after aging and that of the reference, that is, the specific heat scan obtained cooling down the sample to $183 \mathrm{~K}$ and immediately heated up 
at $393 \mathrm{~K}$. This was done using the relation,

$$
\Delta H\left(T_{a}, t_{a}\right)=\int_{T<<T_{g}}^{T>>T_{g}}\left(C_{p}^{a}(T)-C_{p}^{u}(T)\right) d T,
$$

where $C_{p}^{a}(\mathrm{~T})$ and $C_{p}^{u}(\mathrm{~T})$ are the heat capacity measured after the annealing and that of the unannealed sample, respectively.

\section{RESULTS}

Figure 1 shows the specific heat as a function of temperature for $95 \mathrm{~nm}$ thick PS samples after aging at the indicated temperatures for $480 \mathrm{~min}$. The observation of the figure indicates that aging in proximity of the films' $T_{g}$ ( $369 \mathrm{~K}$ after cooling at $20 \mathrm{~K} / \mathrm{min})^{22}$ exhibits the standard features of enthalpy recovery, consisting of a rather narrow endothermic overshoot in proximity of $T_{g}$. Upon lowering the aging temperature, the endothermic overshoot, indicating the presence of recovered enthalpy, first reduces in magnitude, subsequently shifts to lower temperature, and, more importantly, exhibits pronounced broadening. After aging at $T_{a}=253 \mathrm{~K}$, this extends over a temperature interval as large as $\sim 90 \mathrm{~K}$. After aging at even lower temperatures, as shown by the scan corresponding to $T_{a}=233 \mathrm{~K}$, the endothermic overshoot shifts to higher temperatures and becomes again narrower.

Figure 2 shows the enthalpy recovered after $480 \mathrm{~min}$ aging as a function of $T_{a}$. Apart from the analysis of data shown in Fig. 1 for $95 \mathrm{~nm}$ thick films through Equation (1), the recovered enthalpy after $480 \mathrm{~min}$ for $30 \mathrm{~nm}$ thick films and bulk PS is also shown. Data for bulk PS generally agree with those contained in previous reports for various polymers including PS, showing that the aging rate, after reaching a maximum not far from $T_{g}$, exhibits a monotonous decrease at lower temperatures. ${ }^{3,58}$ The inset of Fig. 2 shows the enlargement of the area contained by the dashed line in the main panel. Furthermore, the enthalpy recovered by bulk PS aged for 5 days at different temperatures is also presented in the inset of Fig. 2 (see below for discussion of the latter data). These results indicate that, in the explored time scale, 95 and $30 \mathrm{~nm}$ thick films exhibit two mechanisms of equilibrium recovery, whereas this time scale appears to be too short to evidence such phenomenology

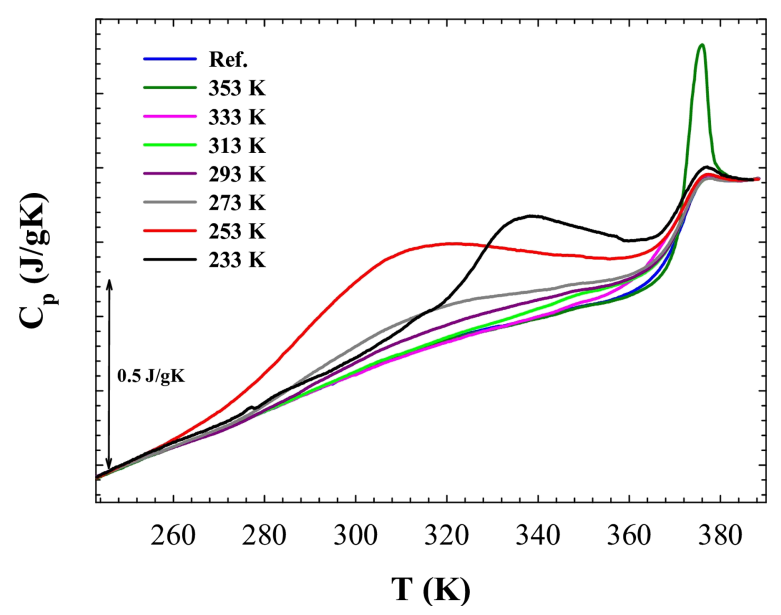

FIG. 1. Heat capacity as a function of temperature for $95 \mathrm{~nm}$ PS films after 480 min aging at the indicated aging temperatures. The reference curve corresponds to a sample heated up just after cooling.

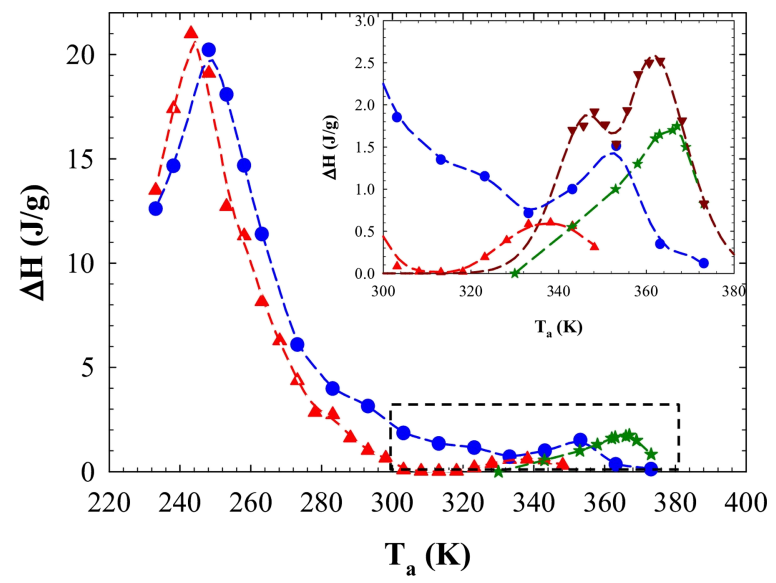

FIG. 2. Recovered enthalpy as a function of the aging temperature after 480 min aging for the following samples: bulk PS (green stars); films with thickness $95 \mathrm{~nm}$ (blue circles) and $30 \mathrm{~nm}$ (red triangles). The latter data are taken from Ref. 48. The inset shows an enlargement of the area delimited by the dashed line of the main panel. Symbols are as in the main panel. Brown inverted triangles are for bulk PS aged for 5 days. The lines are guides to the eye.

in bulk PS. Furthermore, from the observation of Fig. 2, it is interesting to notice that, at the same aging time, the fast mechanism of equilibration allows recovering amounts of enthalpy, which are one order of magnitude larger than those recovered by the slow mechanism in proximity of $T_{g}$. While the massive enthalpy recovery observed in the present study for thin PS films at sub-ambient conditions represents a novel result, the behavior observed at temperatures not too far from $T_{g}$, with a maximum in the recovered enthalpy, is consistent with previous studies, where the physical aging of thin PS films in various configurations was investigated above room temperature. ${ }^{59,60}$

The specific heat as a function of temperature for $95 \mathrm{~nm}$ thick films aged at $353 \mathrm{~K}$ for different annealing times is presented in Fig. 3. As expected, a progressive increase of the endothermic overshoot with aging time can be observed. While the increase of the endothermic overshoot appears to be rather monotonous until $10^{6} \mathrm{~s}$, importantly its increase exhibits a significant enhancement for the longest investigated aging time $\left(4 \times 10^{6} \mathrm{~s}\right)$.

A quantitative analysis of the behavior of equilibrium recovery in isothermal conditions is shown in the inset of Fig. 3, where the amount of recovered enthalpy at $353 \mathrm{~K}$ is shown as a function of the aging time. The observation of such inset indicates that, after reaching a plateau at $\sim 10^{4} \mathrm{~s}$, the behavior of the recovered enthalpy experiences further increase at aging times longer than $\sim 10^{6} \mathrm{~s}$. The inset of Fig. 3 also shows the aging time dependence of the enthalpy recovered at 358 and $363 \mathrm{~K}$. As can be observed, the double step decay is hardly visible at $358 \mathrm{~K}$ due to the incipient merging of the two decays. Furthermore, at $363 \mathrm{~K}$ only one mechanism of recovery is visible.

The enthalpy recovery over a wide temperature range for thin films with thickness 67 and $95 \mathrm{~nm}$ allows the determination of the time scale to reach each plateau, that is, the equilibration time $\tau_{e q}$. This was also done on enthalpy recovery data of $30 \mathrm{~nm}$ thick PS films and bulk PS presented elsewhere. ${ }^{42,48}$ The determination of $\tau_{e q}$ was carried out by means of the geometric construction depicted in Fig. 3. In particular, 


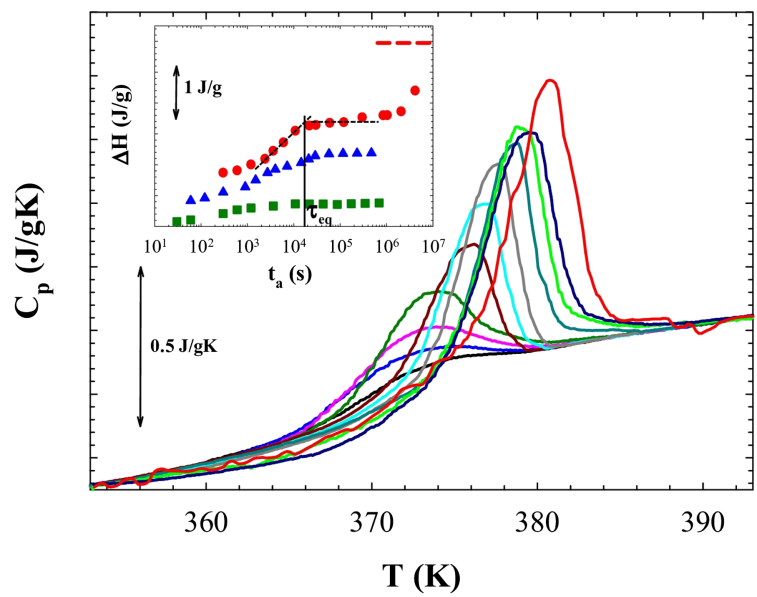

FIG. 3. Specific heat as a function of temperature for 95 thick films aged at $353 \mathrm{~K}$ over the following aging times: $1.2 \times 10^{3} \mathrm{~s}$ (blue); $3.6 \times 10^{3} \mathrm{~s}$ (pink); $1.08 \times 10^{4} \mathrm{~s}$ (green); $3 \times 10^{4}$ (brown); $6 \times 10^{4}$ (light blue); $1.2 \times 10^{5}$ (gray); $3 \times 10^{5}$ (cyan); $10^{6}$ (light green); $2 \times 10^{6}$ (dark blue); $4 \times 10^{6}$ (red); and the reference (black). The inset shows the aging time evolution of the recovered enthalpy at $353 \mathrm{~K}$ (red circles); $358 \mathrm{~K}$ (blue triangles); and $363 \mathrm{~K}$ (green squares). Data for 353 and $358 \mathrm{~K}$ are shifted vertically by 1.5 and $0.75 \mathrm{~J} / \mathrm{g}$, respectively. The dashed line in the inset is the limit of recoverable enthalpy determined extrapolating the melt line to the aging temperature. The error in the determination of the recovered enthalpy is $\pm 0.1 \mathrm{~J} / \mathrm{g}$.

$\tau_{e q}$ was determined from the intersection of the lines drawn fitting linearly data at the time interval corresponding to the plateau and that of maximum slope of the enthalpy change.

In Fig. 4, the temperature dependence of the equilibration times of the fast and the slow mechanisms of recovery is presented. The behavior shown in Fig. 4 suggests that the temperature dependence of the two equilibration times toward equilibrium exhibits activation energies independent of the film thickness. To quantitatively inspect such analogy among films and the bulk, we performed a $\tau_{e q} /$ thickness superposition taking the bulk as the reference. Operationally this implies shifting vertically $\tau_{e q}$ of a factor $a_{h}$. For the fast $\tau_{e q}$, we have superposed data for 67 and $95 \mathrm{~nm}$ on the point at the lowest temperature (largest $\tau_{e q}$ ) of bulk PS. As data related to the fast decay of the $30 \mathrm{~nm}$ thick films are located in a temperature

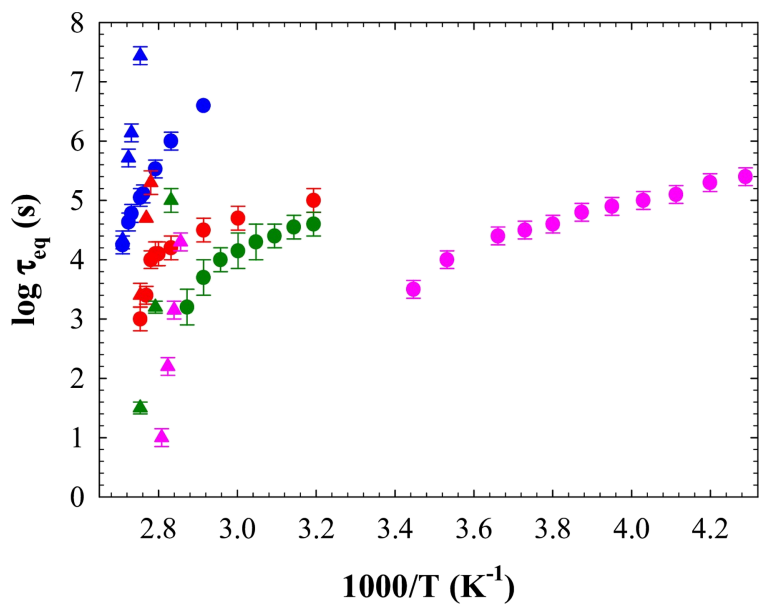

FIG. 4. Logarithm of the equilibration time as a function of the inverse temperature for thin PS films with the following thickness: $30 \mathrm{~nm}$ (pink); $67 \mathrm{~nm}$ (green); $95 \mathrm{~nm}$ (red); and bulk PS (blue). Circles and triangles are for the first and the second decays, respectively.

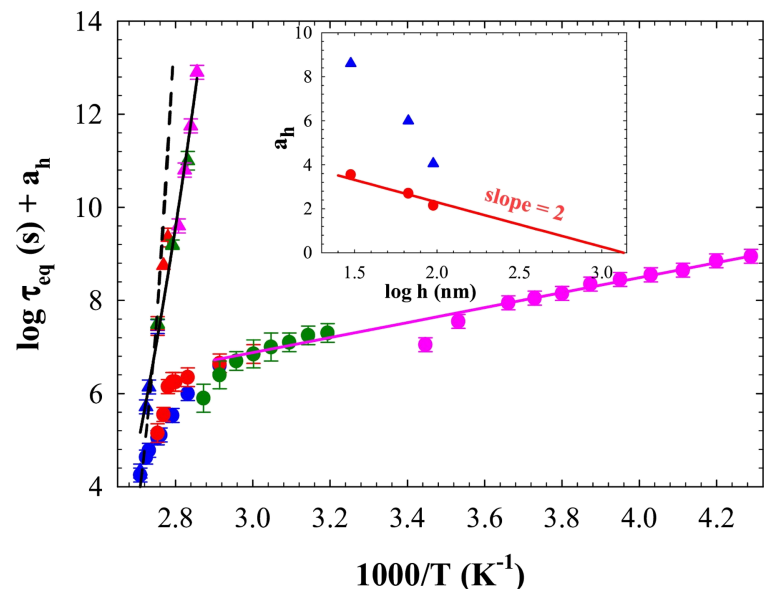

FIG. 5. Logarithm of the equilibration time shifted by an amount, $a_{h}$, allowing superposition with bulk PS $\tau_{e q}$, as a function of the inverse temperature for all investigated systems. Symbols are as in Fig. 4. The pink line and the black continuous and dashed lines are the fits to the Arrhenius and the VFT equations (the former for thin films and the latter for bulk), respectively (see text for details). The inset shows the shift factor for the first (red circles) and second (blue triangles) mechanisms of recovery as a function of the logarithm of the thickness. The red line is the best fit to shift factors related to the first decay imposing a slope of two (see text for details).

range well separated from that of the other systems, the shifting procedure was preceded by the fitting of such data below $273 \mathrm{~K}$ by the Arrhenius law. Superposition of data related to the slow decay was performed minimizing the difference between bulk data and those of the films, in the temperature range where both sets of data are present. The outcome of the superposition procedure is shown in Fig. 5, where the scaled logarithm of the equilibration time related to the first and second decay is presented as a function of the inverse temperature.

In line with the qualitative observation of similar temperature dependence of the $\tau_{e q}$ of both enthalpy recovery mechanism toward equilibrium, the superposition shown in Fig. 5 appears to be accurate. In the inset of Fig. 5, the thickness dependence of the shift factors related to both decays is presented. Inspection of the figure indicates that the shift factor of the fast mechanism of recovery has milder thickness dependence in comparison to that of the slow one.

\section{DISCUSSION}

Altogether, the results presented in Sec. III provide evidence for the presence of a double mechanism of recovery in the equilibrium recovery of thin PS films with thickness ranging from 30 to $95 \mathrm{~nm}$. This result well compares with an analogous study on the enthalpy recovery of bulk PS, also exhibiting two mechanisms of equilibrium recovery. ${ }^{42}$ However, given the well-documented faster evolution toward equilibrium of thin polymer films in comparison to the bulk,,${ }^{14}$ the phenomenology based on the double mechanism is observed in time scales considerably more amenable to the experimental practice. For instance, inspection of Fig. 4 indicates that complete equilibration can be achieved in $30 \mathrm{~nm}$ thick films at temperature as low as $350 \mathrm{~K}$ in a time scale of several hours. Furthermore, the large time scales needed to split the fast and the slow processes in bulk PS ${ }^{42}$ imply that for isochronal experiments with an aging time of $480 \mathrm{~min}$, only a single mechanism 
is visible, that is, one peak in the recovered enthalpy as a function of the aging temperature is observed (see Fig. 2). However, as shown in the inset of Fig. 2, isochronal aging experiments in bulk PS over a time scale as large as 5 days show the presence of two peaks in the annealing temperature dependence of the recovered enthalpy, in analogy with thin PS films.

Given the main outcome of the present study, the challenge is unveiling what are the mechanisms rendering equilibration of thin films orders of magnitude faster than in bulk. Important insight in this sense can be gained from the thickness dependence of the shift factor. As can be observed in the inset of Fig. 5, the shift factor associated with the fast recovery mechanism scales with the logarithm of the thickness with a slope of two. This is compatible with the hypothesis of diffusion of free volume holes toward the free interface as the mechanism responsible for densification. ${ }^{17,61-64}$ Strong experimental evidences supporting this mechanism have been very recently provided following the densification of stable colloidal glasses by Han and co-workers. ${ }^{65}$ They showed that densification occurs via free-volume "bubbles" migrating towards the free surface. Within this framework, the relation $h^{2} \sim D \exp a_{h}$ must be fulfilled, ${ }^{17,64}$ where $D$ is the hole diffusion coefficient of free volume holes. The diffusion mechanism shares the analogy with the propagation of a velocity front in the devitrification of physically vapor deposited (PVD) glasses ${ }^{66-68}$ in that recovery of equilibrium is initiated from the free interface. However, in contrast with the diffusion mechanism providing dependence between the shift factor and the logarithm of the thickness with a slope of two, the mechanism based on the propagation of a constant velocity front rather gives a slope of one.

Conversely, the thickness dependence of the shift factor associated with the slow mechanism of recovery exhibits clear deviations from the exponent two. The inability of conventional diffusion arguments to describe the slow recovery of equilibrium implies that additional ingredients are needed in such a case. One possibility would be the involvement of a slow molecular mechanism with the rate of spontaneous fluctuations depending on the films thickness, at least below $100 \mathrm{~nm}$. Within the hole diffusion model, this would imply assuming a diffusion coefficient increasing with the film thickness decrease. In such a case, a slope larger than two in the shift factor as a function of the logarithm of the thickness would naturally arise. The scenario based on significant modifications of the molecular mobility, as far as the slow molecular mechanism is concerned and only well below the bulk $T_{g}$, agrees with recent theoretical approaches based on the string-like motion 69 and activated glassy relaxation. ${ }^{70}$ Both approaches involve the presence of a growing length scale associated with molecular motion with decreasing temperature. This means that deep in the glassy state, as is the case of enthalpy recovery experiments of the present study, such length scale may be of the same order of the film thickness.

Further details on the distinctive features of the molecular mechanisms of equilibration can be derived considering how rapidly $\tau_{e q}$ varies with the temperature. The continuous lines in Fig. 5 are the fits of shifted experimental $\tau_{e q}$. In particular, regarding the fast mechanism of recovery, the Arrhenius law - with $E_{a}=31 \mathrm{~kJ} / \mathrm{mol}$ - was employed to fit to low temperature data of $30 \mathrm{~nm}$ thick films, and extended to catch low temperature data of larger thicknesses. The most striking feature of the Arrhenius fit to data is the magnitude of the activation energy, $E_{a}$. The value of $E_{a}$ indicates a weakly activated molecular mechanism. This is about five times smaller than the activation energy of PS $\beta$ relaxation $(160 \mathrm{~kJ} / \mathrm{mol}) .^{71}$ This excludes the molecular motion responsible for such process as a candidate for the densification associated with the fast decay. This result could be related to that found in metallic glasses, where, by means of x-ray photon correlation spectroscopy (XPCS), a molecular process with low activation energy was identified. $^{54,55}$

Regarding the temperature dependence of $\tau_{e q}$ associated with the slow process, the continuous line in Fig. 5 is the fit through the Vogel-Fulcher-Tammann (VFT) equation, ${ }^{72-74}$

$$
\log \tau_{e q}+a_{h}=A+\frac{B}{\left(T-T_{0}\right) \ln 10},
$$

where $A$ and $B$ are temperature independent parameters and $T_{0}$ is the Vogel temperature. The fit to scaled equilibration times of Fig. 5 delivers $A=-11.05, B=2920 \mathrm{~K}$, and $T_{0}=274 \mathrm{~K}$. Interestingly, this provides a considerably less steep temperature dependence than that of the VFT behavior of bulk PS obtained fitting data of $\tau_{e q}$ combined with relaxation data obtained by various methods measuring the rate of spontaneous fluctuations ${ }^{5,7,34}$ (dashed line in Fig. 5). In this case, the following VFT parameters provide the best fit to data: $A=-11.5 ; B=1070 \mathrm{~K}$; and $T_{0}=339 \mathrm{~K}$. This result would indicate that deviations from the conventional VFT behavior extrapolated from above the bulk $T_{g}$ toward shorter time scales exist. Several studies provide indications in this sense. ${ }^{75-78}$ Within this framework, the divergence of the relaxation time expected from the conventional VFT equation could be avoided. However, it is worth pointing out that the previous discussion is valid only if the bulk equilibrium dynamics are retained when decreasing the thickness down to $30 \mathrm{~nm}$. As discussed in Section I, this is certainly the case for what concerns the equilibrium dynamics of freestanding films (and therefore stacked films too) ${ }^{18}$ in proximity of the bulk $T_{g}$. On the other hand, as pointed out when discussing the suitability of the model based on free volume holes diffusion, a scenario where the rate of spontaneous fluctuations related to the slow mechanism in films is increased in comparison to the bulk well below the bulk $T_{g}$ can be hypothesized. In this context, it is worth pointing out that both, theoretical approaches ${ }^{79,80}$ as well as experimental data ${ }^{81}$ on extremely long time scales rather support the scenario based on divergent relaxation time of the slow process. These issues are currently matter of ongoing debate. ${ }^{10,82,83}$

Finally, our results indicate that, in appropriate conditions, glasses exhibiting thermodynamic state with low enthalpy can be achieved. For instance, in the present work we show that 30-95 nm PS films aged for as little as several hours undergo an enthalpy decrease from the glass cooled down at standard rates $(20 \mathrm{~K} / \mathrm{min}$ in the present study) as large as more than $20 \mathrm{~J} / \mathrm{g}$, corresponding to a decrease of the fictive temperature $\left(T_{f}\right)$ - that is, the temperature corresponding to the intercept of the extrapolated glass and equilibrium lines for a given glass - as large as $\sim 70 \mathrm{~K} .{ }^{48}$ This corresponds to an increase of the density of approximately $10 \%$. Furthermore, isothermal 
enthalpy recovery experiments on $30 \mathrm{~nm}$ films published elsewhere $^{48}$ show that after aging for a few days at $233 \mathrm{~K}, T_{f}$ as low as the expected Kauzmann temperature ${ }^{84}$ for bulk PS can be achieved. The variation of $T_{f}$ is considerably larger than that normally observed in aging experiments in proximity of the glass $T_{g}$, where a decrease of a few Kelvin is normally observed. This result, apart from the fundamental implications on the solution of the Kauzmann paradox ${ }^{84}$ and the existence of an ideal glass transition ${ }^{85}$ discussed in Ref. 48 , may potentially open new areas of research in those fields where highly dense glasses are required. These include applications in membrane for gas separation, ${ }^{86}$ drug delivery, ${ }^{87}$ and organic electronics, ${ }^{88}$ to name a few of the most explored in recent years.

\section{CONCLUSIONS}

Investigating the enthalpy recovery deep in the glassy state of thin PS films with thickness ranging from 30 to $95 \mathrm{~nm}$, the presence of two mechanisms of equilibration was detected. This result is analogous to that recently found in bulk PS. ${ }^{42}$ However, the time scales involved in the equilibration of thin films are considerably shorter than those needed for bulk PS. Nonetheless, it is possible to scale separately the time to reach equilibrium for each of the two mechanisms of recovery and for all investigated temperatures and thicknesses on a common master curve. This result indicates that the molecular mechanisms involved in the equilibration are thickness independent, though the time scales of equilibrium recovery are different.

Analysis of the thickness dependence of the shift factors reveals that the model based on free volume holes diffusion is compatible with the equilibration associated with the fast mechanism of the recovery. Conversely, equilibration times related to the slow mechanism of recovery need to be shifted considerably more than expected according to simple diffusion arguments.

Further insight is achieved analyzing the temperature dependence of the equilibration times related to the two mechanisms of recovery. The fast one exhibits Arrhenius temperature dependence with activation energy about five times lower than that of PS $\beta$ relaxation. Pronounced temperature dependence of the slow equilibration time is found. However, such dependence appears to be milder than the VFT extrapolation to low temperature of bulk PS data.

\section{SUPPLEMENTARY MATERIAL}

See supplementary material for heat flow scans corresponding to specific heat data shown in Figures 1 and 3, plus those for bulk PS aged at $252 \mathrm{~K}$ for 2 days and $67 \mathrm{~nm}$ thick films in various conditions.

\section{ACKNOWLEDGMENTS}

The authors acknowledge the University of the Basque Country and Basque Country Government (Reference No. IT-654-13 (GV)), Depto. Educación, Universidades e investigación, and Spanish Government (Grant No. MAT201563704-P, (MINECO/FEDER, UE)) for their financial support.
${ }^{1}$ P. G. Debenedetti, Metastable Liquids: Concepts and Principles (Princeton University Press, Princeton, 1996).

${ }^{2}$ T. V. Tropin, J. W. Schmelzer, and V. L. Aksenov, Phys.-Usp. 59, 42 (2016).

${ }^{3}$ L. C. E. Struik, Physical Aging in Amorphous Polymers and Other Materials (Technische Hogeschool Delft, 1977).

${ }^{4}$ A. J. Kovacs, Fortschr. Hochpolym.-Forsch. 3, 394 (1963).

${ }^{5}$ S. Weyer, M. Merzlyakov, and C. Schick, Thermochim. Acta 377, 85 (2001).

${ }^{6}$ L.-M. Wang, V. Velikov, and C. A. Angell, J. Chem. Phys. 117, 10184 (2002).

${ }^{7}$ J. E. K. Schawe, J. Chem. Phys. 141, 184905 (2014).

${ }^{8}$ X. Guo, M. M. Smedskjaer, and J. C. Mauro, J. Phys. Chem. B 120, 3226 (2016).

${ }^{9}$ G. Johari, Thermochim. Acta 523, 97 (2011).

${ }^{10}$ D. Cangialosi, J. Phys.: Condens. Matter 26, 153101 (2014).

${ }^{11}$ M. D. Ediger and J. A. Forrest, Macromolecules 47, 471 (2014).

${ }^{12}$ R. D. Priestley, D. Cangialosi, and S. Napolitano, J. Non-Cryst. Solids 407, 288 (2015).

${ }^{13}$ F. Kremer, M. Tress, and E. U. Mapesa, J. Non-Cryst. Solids 407, 277 (2015).

${ }^{14}$ D. Cangialosi, A. Alegria, and J. Colmenero, Prog. Polym. Sci. 54-55, 128 (2016).

${ }^{15}$ J. L. Keddie, R. A. L. Jones, and R. A. Cory, Europhys. Lett. 27, 59 (1994).

${ }^{16}$ A. Sepúlveda, E. Leon-Gutierrez, M. Gonzalez-Silveira, C. RodríguezTinoco, M. T. Clavaguera-Mora, and J. Rodríguez-Viejo, Phys. Rev. Lett. 107, 025901 (2011).

${ }^{17}$ V. M. Boucher, D. Cangialosi, A. Alegría, and J. Colmenero, Macromolecules 45, 5296 (2012).

${ }^{18}$ K. Paeng, S. F. Swallen, and M. D. Ediger, J. Am. Chem. Soc. 133, 8444 (2011).

${ }^{19}$ H. Yin, S. Madkour, and A. Schoenhals, Macromolecules 48, 4936 (2015).

${ }^{20}$ W. L. Merling, J. B. Mileski, J. F. Douglas, and D. S. Simmons, Macromolecules 49, 7597 (2016).

${ }^{21}$ V. Lupascu, S. J. Picken, and M. Wbbenhorst, J. Non-Cryst. Solids 352, 5594 (2006).

${ }^{22}$ V. M. Boucher, D. Cangialosi, H. Yin, A. Schonhals, A. Alegria, and J. Colmenero, Soft Matter 8, 5119 (2012).

${ }^{23}$ C. Zhang, V. M. Boucher, D. Cangialosi, and R. D. Priestley, Polymer 54, 230 (2013).

${ }^{24}$ C. Mathlouthi, F. Hugenell, F. Delpech, and Y. Rharbi, Macromolecules 50, 472 (2017).

${ }^{25}$ H. Huth, A. A. Minakov, and C. Schick, J. Polym. Sci., Part B: Polym. Phys. 44, 2996 (2006)

${ }^{26}$ M. Tress, M. Erber, E. U. Mapesa, H. Huth, J. Mueller, A. Serghei, C. Schick, K.-J. Eichhorn, B. Voit, and F. Kremer, Macromolecules 43, 9937 (2010).

${ }^{27}$ S. Gao, Y. P. Koh, and S. L. Simon, Macromolecules 46, 562 (2013).

${ }^{28}$ D. Cangialosi, A. Alegría, and J. Colmenero, "Cooling rate dependent glass transition in thin polymer films and in bulk," in Fast Scanning Calorimetry, edited by C. Schick and V. Mathot (Springer International Publishing, Cham, 2016), pp. 403-431.

${ }^{29}$ P. Pfromm and W. Koros, Polymer 36, 2379 (1995).

${ }^{30}$ R. D. Priestley, C. J. Ellison, L. J. Broadbelt, and J. M. Torkelson, Science 309, 456 (2005).

${ }^{31}$ B. W. Rowe, B. D. Freeman, and D. R. Paul, Polymer 50, 5565 (2009).

${ }^{32}$ M. Yavari, T. Le, and H. Lin, J. Membr. Sci. 525, 387 (2017).

${ }^{33}$ X. Monnier, A. Saiter, and E. Dargent, Thermochim. Acta 648, 13 (2017).

${ }^{34}$ V. M. Boucher, D. Cangialosi, A. Alegria, J. Colmenero, I. Pastoriza-Santos, and L. M. Liz-Marzan, Soft Matter 7, 3607 (2011).

${ }^{35}$ V. M. Boucher, D. Cangialosi, A. Alegría, and J. Colmenero, Phys. Rev. E 86, 041501 (2012).

${ }^{36}$ D. Cangialosi, V. M. Boucher, A. Alegria, and J. Colmenero, Soft Matter $\mathbf{9}$ 8619 (2013).

${ }^{37}$ V. Ramakrishnan, S. Harsiny, J. G. P. Goossens, T. L. Hoeks, and G. W. M. Peters, J. Polym. Sci., Part B: Polym. Phys. 54, 2069 (2016).

${ }^{38}$ S. Napolitano, C. Rotella, and M. Wubbenhorst, ACS Macro Lett. 1, 1189 (2012).

${ }^{39}$ S. Napolitano and D. Cangialosi, Macromolecules 46, 8051 (2013).

${ }^{40}$ M. J. Burroughs, S. Napolitano, D. Cangialosi, and R. D. Priestley, Macromolecules 49, 4647 (2016).

${ }^{41}$ V. M. Boucher, D. Cangialosi, A. Alegría, and J. Colmenero, Macromolecules 44, 8333 (2011).

${ }^{42}$ D. Cangialosi, V. M. Boucher, A. Alegría, and J. Colmenero, Phys. Rev. Lett. 111, 095701 (2013).

${ }^{43}$ A. Wisitsorasak and P. G. Wolynes, J. Phys. Chem. B 118, 7835 (2014).

${ }^{44}$ R. Wimberger-Friedl and J. G. de Bruin, Macromolecules 29, 4992 (1996). 
${ }^{45}$ Y. P. Koh, L. Grassia, and S. L. Simon, Thermochim. Acta 603, 135 (2015).

${ }^{46}$ Y. P. Koh, S. Gao, and S. L. Simon, Polymer 96, 182 (2016).

${ }^{47}$ Y. P. Koh and S. L. Simon, Macromolecules 46, 5815 (2013).

${ }^{48}$ V. M. Boucher, D. Cangialosi, A. Alegria, and J. Colmenero, Phys. Chem. Chem. Phys. 19, 961 (2017).

${ }^{49}$ R. S. Miller and R. A. MacPhail, J. Chem. Phys. 106, 3393 (1997).

${ }^{50}$ R. Golovchak, A. Kozdras, V. Balitska, and O. Shpotyuk, J. Phys.: Condens. Matter 24, 505106 (2012).

${ }^{51}$ R. Golovchak, A. Kozdras, O. Shpotyuk, and V. Balitska, J. Phys.: Condens. Matter 28, 355101 (2016).

${ }^{52}$ D. V. Louzguine-Luzgin, I. Seki, T. Yamamoto, H. Kawaji, C. Suryanarayana, and A. Inoue, Phys. Rev. B 81, 144202 (2010).

${ }^{53}$ C. Wang, L. Hu, C. Wei, X. Tong, C. Zhou, Q. Sun, X. Hui, and Y. Yue, J. Chem. Phys. 141, 164507 (2014).

${ }^{54}$ V. M. Giordano and B. Ruta, Nat. Commun. 7, 10344 (2016).

${ }^{55}$ P. Luo, P. Wen, H. Y. Bai, B. Ruta, and W. H. Wang, e-print arXiv:1609.09611.

${ }^{56}$ G. Reiter, M. Hamieh, P. Damman, S. Sclavons, S. Gabriele, T. Vilmin, and E. Raphael, Nat. Mater. 4, 754 (2005).

${ }^{57}$ S. J. Whitlow and R. P. Wool, Macromolecules 24, 5926 (1991).

${ }^{58}$ R. Greiner and F. R. Schwarzl, Rheol. Acta 23, 378 (1984).

${ }^{59}$ J. E. Pye, K. A. Rohald, E. A. Baker, and C. B. Roth, Macromolecules 43, 8296 (2010).

${ }^{60}$ E. Lopez and S. L. Simon, Macromolecules 49, 2365 (2016).

${ }^{61}$ M. McCaig, D. Paul, and J. Barlow, Polymer 41, 639 (2000).

${ }^{62}$ D. Cangialosi, M. Wübbenhorst, J. Groenewold, E. Mendes, H. Schut, A. van Veen, and S. J. Picken, Phys. Rev. B 70, 224213 (2004).

${ }^{63}$ A. W. Thornton and A. J. Hill, Ind. Eng. Chem. Res. 49, 12119 (2010).

${ }^{64}$ V. M. Boucher, D. Cangialosi, A. Alegria, and J. Colmenero, Thermochim. Acta 575, 233 (2014).

${ }^{65}$ X. Cao, H. Zhang, and Y. Han, e-print arXiv:1609.09611.

${ }^{66}$ S. F. Swallen, K. Traynor, R. J. McMahon, M. D. Ediger, and T. E. Mates, Phys. Rev. Lett. 102, 065503 (2009).
${ }^{67}$ M. Ahrenberg, Y. Z. Chua, K. R. Whitaker, H. Huth, M. D. Ediger, and C. Schick, J. Chem. Phys. 138, 024501 (2013).

${ }^{68}$ C. Rodriguez-Tinoco, M. Gonzalez-Silveira, J. Rfols-Rib, A. F. Lopeanda, M. T. Clavaguera-Mora, and J. Rodriguez-Viejo, J. Phys. Chem. B 118, 10795 (2014).

${ }^{69}$ T. Salez, J. Salez, K. Dalnoki-Veress, E. Raphael, and J. A. Forrest, Proc. Natl. Acad. Sci. U. S. A. 112, 8227 (2015).

${ }^{70}$ S. Mirigian and K. S. Schweizer, J. Chem. Phys. 143, 244705 (2015).

${ }^{71}$ N. McCrum, B. Read, and G. Williams, Anelastic and Dielectric Effects in Polymeric Solids (John Wiley, 1967).

${ }^{72}$ H. Vogel, Phys. Z. 22, 645 (1921).

${ }^{73}$ G. S. Fulcher, J. Am. Ceram. Soc. 8, 339 (1925).

${ }^{74}$ G. Tammann and W. Hesse, Z. Anorg. Allg. Chem. 156, 245 (1926).

${ }^{75}$ T. Hecksher, A. I. Nielsen, N. B. Olsen, and J. C. Dyre, Nat. Phys. 4, 737 (2008).

${ }^{76}$ J. C. Mauro, Y. Yue, A. J. Ellison, P. K. Gupta, and D. C. Allan, Proc. Natl. Acad. Sci. U. S. A. 106, 19780 (2009).

${ }^{77}$ J. Zhao, S. L. Simon, and G. B. McKenna, Nat. Commun. 4, 1783 (2013).

${ }^{78}$ E. A. A. Pogna, C. Rodriguez-Tinoco, G. Cerullo, C. Ferrante, J. RodriguezViejo, and T. Scopigno, Proc. Natl. Acad. Sci. U. S. A. 112, 2331 (2015).

${ }^{79}$ G. Adam and J. H. Gibbs, J. Chem. Phys. 43, 139 (1965).

${ }^{80}$ T. R. Kirkpatrick, D. Thirumalai, and P. G. Wolynes, Phys. Rev. A 40, 1045 (1989).

${ }^{81}$ H. Wagner and R. Richert, Polymer 38, 255 (1997).

${ }^{82}$ J. Zhao and G. B. McKenna, J. Chem. Phys. 136, 154901 (2012).

${ }^{83}$ R. Richert, J. Chem. Phys. 139, 137101 (2013).

${ }^{84}$ W. Kauzmann, Chem. Rev. 43, 219 (1948).

${ }^{85}$ J. H. Gibbs and E. A. DiMarzio, J. Chem. Phys. 28, 373 (1958).

${ }^{86}$ D. F. Sanders, Z. P. Smith, R. Guo, L. M. Robeson, J. E. McGrath, D. R. Paul, and B. D. Freeman, Polymer 54, 4729 (2013).

${ }^{87}$ S. D. Allison, J. Pharm. Sci. 97, 2022 (2008).

${ }^{88}$ Y.-Y. Noh, N. Zhao, M. Caironi, and H. Sirringhaus, Nat. Nanotechnol. 2, 784 (2007). 